PROCEEDINGS OF THE

AMERICAN MATHEMATICAL SOCIETY

Volume 138, Number 8, August 2010, Pages 2907-2922

S 0002-9939(10)10305-0

Article electronically published on March 19, 2010

\title{
ON HOLOMORPHIC ISOMETRIC EMBEDDINGS OF THE UNIT DISK INTO POLYDISKS
}

\author{
SUI-CHUNG NG
}

(Communicated by Richard A. Wentworth)

\begin{abstract}
We study the classification of holomorphic isometric embeddings of the unit disk into polydisks. As a corollary of our results, we can give a complete classification when the target is the 2-disk and the 3-disk. We also prove that the holomorphic isometric embeddings between polydisks are induced by those of the unit disk into polydisks.
\end{abstract}

\section{INTRODUCTION}

The study of holomorphic isometries between complex manifolds is a classical topic in complex geometry. For Kähler manifolds, the early work dates back to Calabi's seminal work in 1953 [E.53]. In 2003 [LE03], for the purpose of characterizing modular correspondences among other correspondences in arithmetic geometry, Clozel and Ullmo were led to study holomorphic isometric embeddings of an irreducible bounded symmetric domain $\Omega$ into its product spaces $\Omega^{p}, p \geq 2$, up to scalar constants (the isometric constants). Here all bounded symmetric domains are equipped with their Bergman metrics. As explained in their paper, if the rank of $\Omega$ is at least 2, then it follows from Mok's work on Hermitian metric rigidity that the isometric embeddings are necessarily totally geodesic (i.e. the diagonal embeddings). When the rank is equal to 1 , i.e. $\Omega=\mathbb{B}^{n}$, the methods in Hermitian metric rigidity do not apply. Nevertheless, for $n \geq 2$, Mok in N.b] (see the remark in this section) proved that the embeddings are also totally geodesic. Thus, there remains the case with $n=1$, i.e. $\Omega=\Delta$, when the domain is the unit disk.

For their purpose, Clozel and Ullmo in LE03. showed that an isometric embedding of $\Delta$ into $\Delta^{p}$ is totally geodesic under the assumption that the image is invariant under certain automorphisms of the target polydisk. They then conjectured that the invariance property is not needed (Conjecture 2.2 in [LE03]). However, Mok N.ng constructed a non-totally geodesic isometric embedding of $\Delta$ into $\Delta^{p}$ for each $p \geq 2$, which we will call the $p$-th root embedding. Among the $p$-th root embeddings, together with the diagonal embeddings, we can form compositions or reparametrize them by Möbius transformations to obtain more isometric embeddings of the unit disk into polydisks. The question of whether all isometric embeddings can be obtained in such a way is certainly very interesting.

The purpose of this paper is to show that under certain restrictions on the sheeting number of their algebraic extensions, all isometric embeddings can be

Received by the editors July 30, 2009 and, in revised form, November 27, 2009.

2010 Mathematics Subject Classification. Primary 53C35, 53C55.

(C)2010 American Mathematical Society
Reverts to public domain 28 years from publication 2907 
constructed in this way. As a corollary, we can determine all isometric embeddings of $\Delta$ into $\Delta^{2}$ and $\Delta^{3}$. They are the diagonal embeddings and those embeddings constructed from the square root embedding and the cube root embedding. In the final part of the paper, we will also prove a "splitting theorem", which essentially says that the study of isometric embeddings of $\Delta^{q}$ into $\Delta^{p}$ reduces to the study of isometric embeddings of $\Delta$ into $\Delta^{p}$.

Our work is based on the functional equation

$$
\prod_{i=1}^{p}\left(1-\left|f_{i}\right|^{2}\right)=\left(1-|z|^{2}\right)^{k}
$$

where $z \mapsto\left(f_{1}(z), \ldots, f_{p}(z)\right)$ is an isometric embedding of $\Delta$ into $\Delta^{p}$ with the isometric constant equal to $k$. It is derived from the isometry condition on the Kähler potentials of $\Delta$ and $\Delta^{p}$. From the functional equation it can then be proven that the graph of the embedding extends algebraically and the extension is a finite branched cover over the domain. After establishing the algebraic extension, some general properties of the embedding such as the distribution of poles and the branchings of the component functions can be obtained. These properties then enable us to give classifications under certain restrictions on the sheeting number.

Remark. In [N.b, the main theorem was proven only for dimensions greater than 1 , while it was stated for all dimensions in the article. For the details, please refer to the erratum [N.a of the article.

\section{The FUnCTIONAL EQUATION}

Let $\lambda$ be a positive real number and $\left(M, g_{M}\right),\left(N, g_{N}\right)$ be Kähler manifolds. A map $F: M \longrightarrow N$ is said to be an isometric embedding with the isometric constant $\lambda$ if $F:\left(M, \lambda g_{M}\right) \longrightarrow\left(N, g_{N}\right)$ is an isometric embedding.

Let $F: \Delta \longrightarrow \Delta^{p}, F(z)=\left(f_{1}(z), \ldots, f_{p}(z)\right)$ be an isometric embedding with the isometric constant $\lambda$. So we will be studying isometric embeddings of $\Delta$ into $\Delta^{p}$ up to scalar constants.

Without loss of generality, we may assume that $F(0)=\mathbf{0}$. The Bergman metric on $\Delta$ is given by $d s_{\Delta}^{2}=2 \operatorname{Re}(g d z \otimes d \bar{z})$, where $g=-2 \frac{\partial^{2}}{\partial z \partial \bar{z}} \log \left(1-|z|^{2}\right)$. We can take as Kähler potentials for $d s_{\Delta}^{2}$ and $d s_{\Delta^{p}}^{2}$ the real analytic functions $-2 \log \left(1-|z|^{2}\right)$ and $-2 \sum_{i=1}^{p} \log \left(1-\left|z_{i}\right|^{2}\right)$ respectively. By the assumption $F^{*} d s_{\Delta^{p}}^{2}=\lambda d s_{\Delta}^{2}$ it follows that

$$
-2 \sqrt{-1} \partial \bar{\partial} \sum_{i=1}^{p} \log \left(1-\left|f_{i}\right|^{2}\right)=-2 \lambda \sqrt{-1} \partial \bar{\partial} \log \left(1-|z|^{2}\right) ;
$$

hence

$$
-\sum_{i=1}^{p} \log \left(1-\left|f_{i}\right|^{2}\right)=-\lambda \log \left(1-|z|^{2}\right)+\operatorname{Re} h
$$

for some holomorphic function $h$ on $\Delta$. Since $F(0)=\mathbf{0}$, comparing Taylor expansions we conclude as in Clozel-Ullmo [LE03, that $h \equiv 0$. Therefore we obtain

$$
-\sum_{i=1}^{p} \log \left(1-\left|f_{i}\right|^{2}\right)=-\lambda \log \left(1-|z|^{2}\right)
$$


i.e.

$$
\prod_{i=1}^{p}\left(1-\left|f_{i}\right|^{2}\right)=\left(1-|z|^{2}\right)^{\lambda} .
$$

By the standard polarization technique, we have the polarized functional equation:

$$
\prod_{i=1}^{p}\left(1-f_{i}(z) \overline{f_{i}(w)}\right)=(1-z \bar{w})^{\lambda}
$$

where $(1-z \bar{w})^{\lambda} \equiv e^{\lambda \log (1-z \bar{w})}$.

For the unit disk, the isometric constant $\lambda$ is necessarily an integer. This can be obtained quite easily once the existence of an algebraic extension is established. The following is proven in Mok N.ng.

Theorem 2.1 (Mok). Let $\Omega \Subset \mathbb{C}^{n}$ and $\Omega^{\prime} \Subset \mathbb{C}^{N}$ be bounded symmetric domains in their Harish-Chandra realizations. Let $\lambda$ be any positive real number and $f$ : $\left(\Omega, \lambda d s_{\Omega}^{2}\right) \longrightarrow\left(\Omega^{\prime}, d s_{\Omega^{\prime}}^{2}\right)$ be a germ of holomorphic isometry at $0 \in \Omega$ with $f(0)=0$.

Then, the germ of the graph of $f$ extends to an affine algebraic variety $S^{\#} \subset$ $\mathbb{C}^{n} \times \mathbb{C}^{N}$ such that $S=S^{\#} \cap\left(\Omega \times \Omega^{\prime}\right)$ is the graph of a holomorphic isometric embedding $F: \Omega \longrightarrow \Omega^{\prime}$ extending the germ of the holomorphic map $f$.

From the existence of algebraic extensions, we can prove

Proposition 2.2. Let $\left(\Delta, \lambda d s_{\Delta}^{2}\right) \longrightarrow\left(\Delta^{p}, d s_{\Delta^{p}}^{2}\right)$ be an isometric embedding. Then $\lambda$ is a positive integer.

Proof. By Theorem 2.1, we know that the embedding can be extended across a general point on the unit circle. Let $z_{0}$ be a point on the unit circle at which the embedding can be extended across in a neighborhood. If we consider (2.1) and substitute $w=z_{0}$, then because each factor on the left-hand side can only vanish with an integral order at $z=z_{0}, \lambda$ on the right-hand side must be a positive integer.

Remark. The integrality also follows from a slight variation of the simpler arguments of Mok [N.b] on algebraic extensions.

We will henceforth denote the isometric constant of an embedding of the unit disk into a polydisk by $k$ as in (1.1), suggesting its integrality. It follows easily from Schwarz's lemma that if the target is $\Delta^{p}$, then $k \leq p$ and the embedding is the diagonal embedding when $k=p$. Thus, for isometric embeddings of $\Delta$ into $\Delta^{p}$, the non-trivial cases are those with $k \in\{1, \ldots, p-1\}$.

\section{EXAMPLES}

For each positive integer $p \geq 2$, Mok N.ng constructed a non-totally geodesic isometric embedding of $\Delta$ into $\Delta^{p}$ with $k=1$. The examples are best expressed when we transform the unit disk into the upper half-plane $\mathcal{H}$. For $\tau \in \mathcal{H}$, write $\tau=r e^{i \theta}$ with $r>0$ and $0<\theta<\pi$. We denote the Poincaré metrics of $\mathcal{H}$ and $\mathcal{H}^{p}$ by $d s_{\mathcal{H}}^{2}$ and $d s_{\mathcal{H}^{p}}^{2}$, respectively.

Proposition 3.1 (Mok). Let $p \geq 2$ be a positive integer and $\gamma=e^{i \pi / p}$. Then the map $F:\left(\mathcal{H}, d s_{\mathcal{H}}^{2}\right) \longrightarrow\left(\mathcal{H}^{p}, d s_{\mathcal{H}^{p}}^{2}\right)$ defined by $F(\tau)=\left(\tau^{\frac{1}{p}}, \gamma \tau^{\frac{1}{p}}, \ldots, \gamma^{p-1} \tau^{\frac{1}{p}}\right)$ is an isometric embedding, where $\tau^{\frac{1}{p}}=r^{\frac{1}{p}} e^{\frac{i \theta}{p}}$. 
This embedding will be called the $p$-th root embedding.

Given an isometric embedding $F: \Delta \longrightarrow \Delta^{p}$, we can construct a family of isometric embeddings parametrized by $\alpha \in \Delta$ and defined by

$$
F^{\alpha}(w)=T\left(F\left(\frac{w+\alpha}{1+\bar{\alpha} w}\right)\right),
$$

where $T \in \operatorname{Aut}\left(\Delta^{p}\right)$ with $T(F(\alpha))=\mathbf{0} \in \Delta^{p}$ such that we have $F^{\alpha}(0)=\mathbf{0}$. $F^{\alpha}$ will be called a reparametrization of $F$.

Besides reparametrizations, we can also form "compositions" among isometric embeddings. More explicitly, for any two isometric embeddings, $F: \Delta \longrightarrow \Delta^{p}$ and $G: \Delta \longrightarrow \Delta^{q}$, they induce an isometric embedding $H: \Delta \longrightarrow \Delta^{p} \longrightarrow$ $\Delta^{p+q-1}$ given by $H=G^{\#} \circ F$, where $G^{\#}: \Delta^{p} \longrightarrow \Delta^{p+q-1}$ and $G^{\#}\left(z_{1}, \ldots, z_{p}\right)=$ $\left(z_{1}, \ldots, z_{p-1}, G\left(z_{p}\right)\right)$. Thus, we have already acquired a great bunch of isometric embeddings by the above constructions from the $p$-th root embeddings. We will prove that these constructions exhaust all isometric embeddings of $\Delta$ into $\Delta^{2}$ and $\Delta^{3}$.

\section{Algebraic extension}

Although the algebraic extension has already been established in Theorem 2.1, we here give a more elementary proof under the assumption that the isometric constant is an integer. Let $F: U \longrightarrow \Delta^{p}$ be a germ of an isometric embedding with the isometric constant $k$, where $U \subset \Delta$ is an open neighborhood containing the origin and $F(0)=\mathbf{0}$. We will assume that every component function is nonconstant. We regard $\Delta$ as a domain in $\mathbb{P}^{1}$. We identify $\mathbb{P}^{1}$ as $\mathbb{C} \cup\{\infty\}$ and use the inhomogeneous coordinate.

Lemma 4.1. Let $f, g: \Omega \subset \mathbb{C}^{m} \longrightarrow \mathbb{C}^{n}, f=\left(f_{1}, \ldots, f_{n}\right), g=\left(g_{1}, \ldots, g_{n}\right)$ be holomorphic maps such that $\|f\|^{2}=\|g\|^{2}$. Then there exists a unitary transformation $U$ in $\mathbb{C}^{n}$ such that $U \circ f=g$.

Proof. Let $f_{j}(Z)=\sum_{|I|=0}^{\infty} f_{j I} Z^{I}$ and $g_{j}(Z)=\sum_{|I|=0}^{\infty} g_{j I} Z^{I}$, where $I$ is a multi-index.

Suppose

$$
\left|f_{1}\right|^{2}+\cdots+\left|f_{n}\right|^{2}=\left|g_{1}\right|^{2}+\cdots+\left|g_{n}\right|^{2} .
$$

Polarizing the power series expansion of the equation, we get

$$
f_{1 I} \overline{f_{1 J}}+\cdots+f_{n I} \overline{f_{n J}}=g_{1 I} \overline{g_{1 J}}+\cdots+g_{n I} \overline{g_{n J}}
$$

for every $I, J \in \mathbb{N}^{m}$.

Let $\mathbf{f}_{I}=\left(f_{1 I}, \ldots, f_{n I}\right)$ and $\mathbf{g}_{I}=\left(g_{1 I}, \ldots, g_{n I}\right)$. Then for the two families $\left\{\mathbf{f}_{I}\right\}_{I \in \mathbb{N}^{m}}$ and $\left\{\mathbf{g}_{I}\right\}_{I \in \mathbb{N}^{m}}$ of vectors in $\mathbb{C}^{n}$, we know that there is an $n \times n$ unitary matrix $U$ such that $U \mathbf{f}_{I}=\mathbf{g}_{I}, \forall I \in \mathbb{N}^{m}$. Hence,

$$
U \sum_{|I|=0}^{\infty} \mathbf{f}_{I} z^{I}=\sum_{|I|=0}^{\infty} \mathbf{g}_{I} z^{I} \Longleftrightarrow U \circ f=g .
$$

Proposition 4.2. There exists an irreducible one-dimensional projective algebraic subvariety $V$ in $\mathbb{P}^{1} \times\left(\mathbb{P}^{1}\right)^{p}$ extending the graph of $F$. In addition, by considering the projection map from $V$ onto the first factor, $V$ is a finite branched cover over $\mathbb{P}^{1}$. 
Proof. $f_{1}, \ldots, f_{p}$ satisfy the functional equation

$$
\prod_{i=1}^{p}\left(1-\left|f_{i}\right|^{2}\right)=\left(1-|z|^{2}\right)^{k}
$$

Expand the brackets and rearrange the above equation to a form

$$
\sum_{j=0}^{d}\left|A_{j}\right|^{2}=\sum_{j=0}^{e}\left|B_{j}\right|^{2},
$$

where $A_{j}, B_{j}$ are analytic functions and $A_{i}=f_{i}$ for $1 \leq i \leq p$. Here $e+1=d=$ $2^{p-1}+\frac{k}{2}$ if $k$ is even and $d=e=2^{p-1}+\frac{k-1}{2}$ if $k$ is odd. If $k$ is even, we add a term $B_{d}:=0$ to the right-hand side making both sides have the same number of terms. Then for each $k$, Lemma 4.1 says that there exists a $d \times d$ unitary matrix U such that

$$
\left(\begin{array}{c}
A_{1} \\
A_{2} \\
\vdots \\
A_{d}
\end{array}\right)=\mathbf{U}\left(\begin{array}{c}
B_{1} \\
B_{2} \\
\vdots \\
B_{d}
\end{array}\right) .
$$

Among the above $e$ equations, the first $p$ of them are of the form

$$
f_{i}=Q_{i}\left(z, f_{1}, \ldots, f_{p}\right), \quad i=1, \ldots, p
$$

where the $Q_{i}$ are polynomials in which every term is either an odd power of $z$ or a product of an even number of the factors $f_{i}$. Applying the Implicit Function Theorem at the point $\mathbf{0} \in \mathbb{P} \times\left(\mathbb{P}^{1}\right)^{p}$, we see that the projective algebraic subvariety defined by these $p$ equation is smooth at $\mathbf{0}$. If we let $V$ be the irreducible component of this subvariety containing $\mathbf{0}$, then $V$ is one-dimensional. It is clear that the germ of the graph of $F$ is contained in $V$. Denote by $\pi$ the projection map from $\mathbb{P}^{1} \times(\mathbb{P})^{p}$ to the first factor. By the Proper Mapping Theorem, $\pi(V)=\mathbb{P}^{1}$ and since $V$ is connected, there exists an integer $n$ such that for a generic point $z \in \mathbb{P}^{1}$, $\#\left(\pi^{-1}(z)\right)=n$.

Let the sheeting number of $V$ relative to $\pi$ be $n$. Consider the projection maps $P_{i}: V \longrightarrow \mathbb{P}^{1} \times \mathbb{P}^{1}$ defined by $P_{i}\left(z, \xi_{1}, \ldots, \xi_{p}\right)=\left(z, \xi_{i}\right)$. Then $V_{i}=P_{i}(V) \subset \mathbb{P}^{1} \times \mathbb{P}^{1}$ is a one-dimensional projective algebraic subvariety containing the germ of the graph of the component function $f_{i}: U \longrightarrow \Delta$. If we also define $\pi_{i}: V_{i} \longrightarrow \mathbb{P}^{1}$ to be the projection map to the first factor, then $\pi$ can be factorized as $\pi=\pi_{i} \circ P_{i}$, and therefore we see that $V_{i}$ is a finite branched cover over $\mathbb{P}^{1}$ with the sheeting number being a factor of $n$.

We are going to show that the defining equation of every $V_{i}$ takes a very specific form. At first, note that there exists a discrete subset $A \subset \mathbb{P}$ such that when restricting to $V \backslash \pi^{-1}(A), \pi$ is a holomorphic covering map. This is obvious if $V$ is smooth and in the general case we can pass to its normalization.

Lemma 4.3. Let $\left(z, a_{1}, \ldots, a_{p}\right),\left(w, b_{1}, \ldots, b_{p}\right)$ be any two points on $V$. Then

$$
\prod_{i=1}^{p}\left(1-a_{i} \overline{b_{i}}\right)=(1-z \bar{w})^{k} .
$$


Proof. We first consider the polarized functional equation

$$
\prod_{i=1}^{p}\left(1-f_{i}(z) \overline{f_{i}(w)}\right)=(1-z \bar{w})^{k}
$$

in a small neighborhood $U \subset \Delta$ of the origin, where $z, w \in U$. We can fix $z$ and do analytic continuation on the variable $w$ and vice versa. Therefore (4.1) is true for all the points on $V \backslash \pi^{-1}(A)$. But $V \backslash \pi^{-1}(A)$ is an open dense subset of $V$ and therefore (4.1) is true for any two points on $V$.

Lemma 4.4. Let $\left(z, a_{1}, \ldots, a_{p}\right)$ be a point on $V$. Then $a_{i}$ is finite for all $i$ if $z$ is finite, i.e. $z \in \mathbb{C} \subset \mathbb{P}^{1} \Longrightarrow\left(a_{1}, \ldots, a_{p}\right) \in \mathbb{C}^{p} \subset\left(\mathbb{P}^{1}\right)^{p}$.

Proof. Suppose $\left(z, a_{1}, \ldots, a_{p}\right)$ is a point on $V$ with $z \in \mathbb{C}$ and $a_{1}=\infty$. Let $U \subset \mathbb{C} \backslash A$ be an open set such that $\pi^{-1}(U)=\bigcup_{l=1}^{n} W_{l}$ and the $W_{l}$ are disjoint. Taking any of the $W_{l}$, say $W_{1}$, for all $\left(w, f_{1}, \ldots, f_{p}\right) \in W_{1}$, we have from (4.1),

$$
\prod_{i=1}^{p}\left(1-a_{i} \overline{f_{i}}\right)=(1-z \bar{w})^{k} .
$$

Now the right-hand side is always finite, and because $a_{1}=\infty$, we have either $f_{1}(w) \equiv 0$ or $\left(1-a_{j} \overline{f_{j}}\right) \equiv 0$ for some $j$. Both will imply that one component function is constant and hence a contradiction.

We fix an $i \in\{1, \ldots, p\}$ and write $h=f_{i}$. We also denote $V_{i}$ by $V_{h}$ and $\pi_{i}$ by $\pi_{z}$

Proposition 4.5. Let the sheeting number of $V_{h}$ relative to $\pi_{z}$ be q. Then $V_{h} \subset$ $\mathbb{P}^{1} \times \mathbb{P}^{1}$ is defined by the equation

$$
h^{q}+P_{q-1}(z) h^{q-1}+P_{q-2}(z) h^{q-2}+\cdots+P_{1}(z) h+P_{0}(z)=0,
$$

where $\forall j, P_{j}(z)=a_{j} z+b_{j}, a_{j}, b_{j} \in \mathbb{C}$ and $b_{0}=0$.

Proof. There exists a discrete subset $A \subset \mathbb{P}^{1}$ such that $\pi_{z}$ is a covering map when restricting to $\pi_{z}^{-1}\left(\mathbb{P}^{1} \backslash A\right)$. We consider the elementary symmetric functions of $h$ relative to the covering map $\pi_{z}$, and they will be meromorphic functions on $\mathbb{P}^{1} \backslash A$. Since $h$ is just a coordinate in $\mathbb{P}^{1} \times \mathbb{P}^{1}$, we see that these elementary symmetric functions can be extended to the whole $\mathbb{P}^{1}$, and hence they are rational functions. Therefore the defining equation of $V_{h}$ is of the form

$$
h^{q}+P_{q-1}(z) h^{q-1}+\cdots+P_{1}(z) h+P_{0}(z)=0,
$$

where $P_{1}, \ldots, P_{q-1}$ are rational functions. We are now going to show that every $P_{i}$ must in fact be a linear polynomial in $z$. First of all, by the Implicit Function Theorem, for a generic choice of $\left(z_{0}, h_{0}\right) \in V_{h}$ with $\left|h_{0}\right|=1$, there exists a neighborhood $U_{0} \subset \mathbb{C}$ containing $h_{0}$ such that $z$ can be expressed as a holomorphic function of $h$. From the functional equation, we see that for $h \in U_{0}$ with $|h|=1$, we have $|z|=1$. Therefore the Schwarz reflection principle implies that

$$
z\left(\frac{1}{\bar{h}}\right)=\frac{1}{\overline{z(h)}}
$$

for $h, 1 / \bar{h} \in U_{0}$. This means that $(z, h)$ satisfies (4.3) if and only if $(1 / \bar{z}, 1 / \bar{h})$ does. Let $r$ be the maximum of the degrees of all the $P_{i}$. Suppose $r>1$. Then for a generic choice of $h^{\#} \in U_{0}$ we can solve (4.3) (when $h=h^{\#}$ ) with $r$ distinct solutions of $z_{1}, \ldots, z_{r}$ such that $\left(z_{1}, h^{\#}\right), \ldots,\left(z_{r}, h^{\#}\right)$ do not lie in the singular part 
of $V_{h}$. Due to the Schwarz reflection principle, when $h=1 / \overline{h^{\#}}$, the solutions of (4.3) are $1 / \bar{z}_{1}, \ldots, 1 / \bar{z}_{r}$ and $\left(1 / \bar{z}_{1}, 1 / \overline{h^{\#}}\right), \ldots,\left(1 / \bar{z}_{r}, 1 / \overline{h^{\#}}\right)$ are regular points of $V_{h}$. Now we can make a reparametrization of the isometric embedding such that $\left(z_{1}, h^{\#}\right) \mapsto(0,0)$. Then $1 / \overline{h^{\#}} \mapsto \infty$ and that means $h$ takes $\infty$ on $r$ distinct points on $\mathbb{P}^{1}$, which contradicts Lemma 4.4. Hence $r$ must be equal to 1. Furthermore, due to Lemma 4.4 again, $P_{j}(z)$ cannot have poles in $\mathbb{C}$ and therefore they are linear polynomials in $z$. Finally, $b_{0}=0$ follows from $h(0)=0$ and the proof is complete.

Corollary 4.6. For every $i$, there exists a rational function $R_{i}$ such that $z=$ $R_{i}\left(f_{i}(z)\right)$. Furthermore, $R_{i}: \mathbb{P}^{1} \longrightarrow \mathbb{P}^{1}$ maps the unit circle into the unit circle.

Corollary 4.7. (4.2) is invariant under the transformation $(z, h) \mapsto(1 / \bar{z}, 1 / \bar{h})$. In particular, for $|z|=1$, if $h_{j}, 1 \leq j \leq q$, are the $q$ roots of (4.2), then $1 / \overline{h_{j}}$, $1 \leq j \leq q$, is a permutation of the roots.

Definition 4.8. The sheeting number of a component function $f_{i}$ is defined to be the degree of $R_{i}$ in Corollary 4.6 .

From the results of Clozel-Ullmo [LE03] or Mok N.ng, every germ of an isometric embedding of the unit disk into a polydisk can be extended over the whole unit disk. But $\mathbb{P}^{1} \backslash \bar{\Delta}$ is biholomorphic to the unit disk, so we conclude that the branch points of $h$ must lie on the unit circle. Let $z_{1}, \ldots, z_{m}$ be the branch points of $h$ and $b_{i}$ the branching order at $z_{i}$. Then from the Riemann-Hurwitz formula, we have

$$
\sum_{i=1}^{m} b_{i}=2(q-1) .
$$

The branching orders $b_{i}$ will be important for classifying isometric embeddings. For example, we will use the following lemma later.

Lemma 4.9. Let $h$ be a component function of an isometric embedding of $\Delta$ into $\Delta^{p}$ and let its sheeting number be $q$. If $h$ has exactly two branch points, then up to reparametrizations, $h$ is a component function of the $q$-th root embedding.

Proof. Since the sheeting number of $h$ is $q$, the branching order at each branch point is $q-1$. Also, we know that the branch points of $h$ lie on the unit circle. Now, by Cayley transformations, we can consider $h$ as a map from the upper halfplane into the upper half-plane. By Corollary 4.6, there is a rational function $R$ of degree $q$ such that $\tau=R(h(\tau))$. We can assume that the branch points of $h$ are 0 and $\infty$ by doing some reparametrization. Then we have $R(h)=\alpha h^{q}$, where $\alpha \in \mathbb{C}$. By Corollary 4.6 again, $R$ maps the real axis into the real axis; therefore $\alpha \in \mathbb{R}$. We can further make the coordinate transformation $h \mapsto|\alpha|^{1 / q} h$ and now we have $\tau=h^{q}$ or $\tau=-h^{q}$. These two equations define $2 q$ branches of an analytic function from the upper half-plane into the complex plane. Among them there are $q$ of them mapping the upper half-plane into the upper half-plane, and they are exactly the $q$ component functions of the $q$-th root embedding.

\section{Sheeting Numbers}

We now return to the isometric embedding itself. For all $i$, let $s_{i}$ be the sheeting number of $f_{i}$. Let us introduce the notation $\Delta^{+}=\Delta$ and $\Delta^{-}=\mathbb{P}^{1} \backslash \bar{\Delta}$. By Corollary 4.6. for each $i$, there exists a rational function $R_{i}$ of degree $s_{i}$ such that 
$z=R_{i}\left(f_{i}(z)\right)$ and $R_{i}$ maps the unit circle into the unit circle. As just mentioned, the branch points of the algebraic extension of the map must lie on the unit circle. Therefore we have $\pi_{i}^{-1}\left(\Delta^{+}\right)=\bigcup_{j=1}^{s_{i}} U_{j ; i} \subset \mathbb{P}^{1} \times \mathbb{P}^{1}, U_{j ; i}$ are disjoint and each lies entirely in either $\Delta^{+} \times \Delta^{+}$or $\Delta^{+} \times \Delta^{-}$.

Similarly we also have $\pi^{-1}\left(\Delta^{+}\right)=\bigcup_{j=1}^{n} U_{j} \subset \mathbb{P}^{1} \times\left(\mathbb{P}^{1}\right)^{p}$, where the $U_{j}$ are disjoint and $n$ is the sheeting number of $V$. We can associate a "parity" to each $U_{j}$, defined as $\left(\chi_{j ; 1}, \ldots, \chi_{j ; p}\right), \chi_{j ; i} \in\{+,-\}$ when $P_{i}\left(U_{j}\right) \subset \Delta^{+} \times \Delta^{\chi_{j ; i}}$.

Lemma 5.1. If $U_{i}$ and $U_{j}$ have the same parity, then $U_{i}=U_{j}$.

Proof. Let the parity of $U_{i}$ and $U_{j}$ be $(+, \ldots,+)$. We can consider $U_{i}$ and $U_{j}$ as the graphs of two isometric embeddings of $\Delta$ into $\Delta^{p}$, denoted by $F_{i}$ and $F_{j}$. From the functional equation, we see that $F_{i}(0)=F_{j}(0)=\mathbf{0} \in\left(\Delta^{+}\right)^{p}$. Thus, $F_{i}$ and $F_{j}$ agree at the origin. However, every point in the unit disk can become the origin by reparametrization. More explicitly, for every $\alpha \in \Delta^{+}$, we consider the reparametrization of $F_{i}$ given by $F_{i}^{\alpha}(w)=T\left(F_{i}\left(\frac{w+\alpha}{1+\bar{\alpha} w}\right)\right)$, where $T$ is an automorphism of $\left(\Delta^{+}\right)^{p}$ such that $T\left(F_{i}(\alpha)\right)=\mathbf{0}$. We can find a path $\gamma:[0,1] \longrightarrow \mathbb{P}^{1}$ with $\gamma(0)=\gamma(1)=0$ such that when we perform the analytic continuation $F_{i}$ along $\gamma$, we will come up with $F_{j}$ at the end. We can correspondingly perform the analytic continuation of $F_{i}^{\alpha}$ along $\gamma^{\alpha}$, where $\gamma^{\alpha}$ is the image of $\gamma$ under the transformation $z=\frac{w+\alpha}{1+\bar{\alpha} w}$. Denote by $\widetilde{F_{i}^{\alpha}}$ the resulting extension. Then we have $\widetilde{F_{i}^{\alpha}}(w)=T\left(F_{j}\left(\frac{w+\alpha}{1+\bar{\alpha} w}\right)\right)$. Observe that $\widetilde{F_{i}^{\alpha}}(0)=\mathbf{0}$ because $\widetilde{F_{i}^{\alpha}}\left(\Delta^{+}\right) \subset\left(\Delta^{+}\right)^{p}$ and $\widetilde{F_{i}^{\alpha}}$ satisfies the functional equation. Hence $T\left(F_{j}(\alpha)\right)=\mathbf{0} \Longrightarrow F_{i}(\alpha)=F_{j}(\alpha)$. Since $\alpha$ is arbitrary, this implies that $U_{i}=U_{j}$. By doing coordinate transformations $(z \mapsto 1 / w)$, the same is true for other parities.

Among the $2^{p}$ different parities, we see that there are only $2^{p-1}$ of them consistent with the functional equation. Hence we have

Proposition 5.2. The sheeting number of $V$ relative to $\pi$ is at most $2^{p-1}$, i.e. $n \leq 2^{p-1}$.

Remark. The maximum $2^{p-1}$ is attainable by composing the square root embedding. More precisely, consider the isometric embedding of $\Delta$ into $\Delta^{p}$ given by $F=F_{p-1} \circ F_{p-2} \circ \cdots \circ F_{1}$, where $F_{1}: \Delta \longrightarrow \Delta^{2}, F(z)=(\alpha(z), \beta(z))$ is the square root embedding and $F_{i}: \Delta^{i} \longrightarrow \Delta^{i+1}$ is defined by $F\left(z_{1}, \ldots, z_{i}\right)=$ $\left(z_{1}, \ldots, z_{i-1}, \alpha\left(z_{i}\right), \beta\left(z_{i}\right)\right)$. Then the sheeting number of the graph of $F$ is $2^{p-1}$.

It follows from Proposition 4.5 that each $f_{i}$ takes $\infty$ on one and only one branch among its $s_{i}$ branches. This observation leads to the following.

Proposition 5.3. The sheeting numbers $s_{i}$ satisfy

$$
\sum_{i=1}^{p} \frac{1}{s_{i}}=k, \quad s_{i} \mid n, \forall i
$$

Proof. As noted after Proposition 4.2 $s_{i}$ is a factor of $n$. Within the $n$ branches of $F$, each coordinate function $f_{i}$ has $s_{i}$ distinct branches and each distinct branch repeats itself $n / s_{i}$ times. Among the $s_{i}$ distinct branches, there is only one branch such that $f_{i}(\infty)=\infty$, and hence $f_{i}$ takes an infinite value $n / s_{i}$ times at infinity within the $n$ branches of $F$. It is easy to deduce from the polarized functional equation that there are exactly $k$ component functions taking infinite value at infinity on each branch of $F$. Therefore the sum of $n / s_{i}$ over $i$ is equal to $k n$. 
From Proposition 5.3, we obtain a lower bound for $n$.

Corollary 5.4. The sheeting number of $V$ relative to $\pi$ is greater than or equal to $p / k$, i.e. $n \geq p / k$.

Proof. Write (5.1) as

$$
\sum_{i=1}^{p} \frac{n}{s_{i}}=n k, \quad s_{i} \mid n, \forall i .
$$

Since $n / s_{i} \geq 1$, we have $n k \geq p$.

\section{TWO THEOREMS}

In this section, we will prove two theorems about the classification of isometric embeddings of the unit disk into polydisks with the isometric constant $k=1$. We will continue to use $V$ to denote the irreducible projective algebraic subvariety in $\mathbb{P}^{1} \times\left(\mathbb{P}^{1}\right)^{p}$ extending the germ of an isometric embedding of $\Delta$ into $\Delta^{p}$. Denote also by $\pi$ the projection map onto the first factor and $n$ the sheeting number of $V$ relative to $\pi$. By Proposition 5.2 and Corollary [5.4 we have $p \leq n \leq 2^{p-1}$. The two theorems are on the extremal cases.

6.1. On the minimal case. Let $p$ be an odd number. Let $F: \Delta \longrightarrow \Delta^{p}, F(z)=$ $\left(f_{1}(z), \ldots, f_{p}(z)\right)$ be an isometric embedding with the isometric constant $k=1$ and $n=p$. From Proposition 5.3, we deduce that the sheeting number of every $f_{i}$ is $p$. In what follows, a complex number of modulus 1 will be called unimodular.

If $z_{0} \in \partial \Delta$ is not a branch point of any $f_{i}$, then there exists a small neighborhood $U$ of $z_{0}$ containing no branch points and such that $\pi^{-1}(U)=\bigcup_{j=1}^{p} U_{j}$, where $U_{j}$ are disjoint and each $U_{j}$ can be regarded as the graph of a branch of the embedding over $U$. Let us denote the $p$ branches of the embedding by $F^{j}(z)=\left(f_{1}^{j}, \ldots, f_{p}^{j}\right)$, $1 \leq j \leq p$.

Lemma 6.1. Let $z_{0} \in \partial \Delta$ such that it is not a branch point of any component function. For each $j$, among the set $\left\{f_{i}^{j}\left(z_{0}\right): 1 \leq i \leq p\right\}$, there is exactly one unimodular value.

Proof. Consider the polarized functional equation

$$
\prod_{i=1}^{p}\left(1-f_{i}^{j}(z) \overline{f_{i}^{j}(w)}\right)=1-z \bar{w}
$$

where $z, w \in U$. Substituting $w=z_{0}$, we get

$$
\prod_{i=1}^{p}\left(1-f_{i}^{j}(z) \overline{f_{i}^{j}\left(z_{0}\right)}\right)=1-z \overline{z_{0}} .
$$

Since the order of zero at $z=z_{0}$ is 1 on the right-hand side and hence on the left-hand side, we therefore obtain the desired result.

Lemma 6.2. Let $z_{0} \in \partial \Delta$ such that it is not a branch point of any component function. For each $i$, among the set $\left\{f_{i}^{j}\left(z_{0}\right): 1 \leq j \leq p\right\}$, there is exactly one unimodular value. In particular, the rational function $R_{i}: \mathbb{P}^{1} \longrightarrow \mathbb{P}^{1}$ in Corollary 4.6 satisfying $z=R_{i}\left(f_{i}(z)\right)$ maps the unit circle bijectively onto the unit circle. 
Proof. By Corollary 4.7, $r \in\left\{f_{i}^{j}\left(z_{0}\right): 1 \leq j \leq p\right\}$ if and only if $1 / \bar{r} \in\left\{f_{i}^{j}\left(z_{0}\right): 1 \leq\right.$ $j \leq p\}$. Since $p$ is odd, at least one element of $\left\{f_{i}^{j}\left(z_{0}\right): 1 \leq j \leq p\right\}$ is unimodular. Combining with Lemma 6.1, we see that exactly one of them is unimodular.

Now we take an arbitrary point $z^{\prime} \in \partial \Delta$. If it happens that $z^{\prime}$ is a branch point of some $f_{i}$, then we cannot define branches of the embedding as above. On the other hand, since each component function $f_{i}$ satisfies an equation of the form of (4.2), so we can still talk about the $p$ "values" of $f_{i}\left(z^{\prime}\right)$ by taking them as the $p$ roots of (4.2) when $z=z^{\prime}$. For each $i$, let $\beta_{l ; i}, 1 \leq l \leq r_{i}$, be the distinct values of $f_{i}\left(z^{\prime}\right)$ and $n_{l ; i}, 1 \leq l \leq r_{i}$, be the corresponding multiplicities.

Lemma 6.3. The number $r_{i}$ and the set $\left\{n_{l ; i}: 1 \leq l \leq r_{i}\right\}$ are independent of $i$.

Proof. We fix an $f_{i}$ and take a sufficiently small neighborhood $U^{\prime}$ of $z^{\prime}$ such that $U^{\prime}$ does not contain any branch point of any component function other than $z^{\prime}$. Recall the notation that $\pi_{i}: V_{i} \longrightarrow \mathbb{P}^{1}$ is the projection map onto the first factor for the graph of $f_{i}$. Since $f_{i}$ is the inverse of a rational function, by taking $U^{\prime}$ sufficiently small we have $\pi_{i}^{-1}\left(U^{\prime}\right)=\bigcup_{l=1}^{r_{i}} W_{i}^{l}$, where $W_{i}^{l}$ are the connected components of $\pi_{i}^{-1}\left(U^{\prime}\right)$ and each $W_{i}^{l}$ is biholomorphic to some open neighborhood of the graph of $\zeta=\xi^{n_{l ; i}}$ at the origin in $\mathbb{C}^{2}$, where $(\zeta, \xi)$ are the coordinates in $\mathbb{C}^{2}$. Now take two component functions $f_{i}$ and $f_{j}$. Since $V, V_{i}$ and $V_{j}$ are all $p$-sheeted covers over $\mathbb{P}^{1}$, the projection maps $P_{i}: V \longrightarrow V_{i}$ and $P_{j}: V \longrightarrow V_{j}$ are both generically one to one and fibre preserving. Hence, there is also a generically one to one and fibre preserving map between $V_{i}$ and $V_{j}$, in particular, between $\pi_{i}^{-1}\left(U^{\prime}\right)$ and $\pi_{j}^{-1}\left(U^{\prime}\right)$. We therefore conclude that $r_{i}=r_{j}$ and after rearranging the index $l$ if necessary, $n_{l ; i}=n_{l ; j}$ for every $l$.

In other words, the branching loci of all the component functions are the same, and we do not need to refer to a specific component function when we speak of branch points. Furthermore, if for each $i$, we let $R_{i}$ be the rational function such that $z=R_{i}\left(f_{i}(z)\right)$, then for any point $\left(z, a_{1}, \ldots, a_{p}\right)$ on $V$, the ramification orders of $R_{i}$ at $a_{i}, 1 \leq i \leq p$, are the same. Thus, we can define the ramification order of $\pi$ at a point $\left(z, a_{1}, \ldots, a_{p}\right) \in V$ to be the ramification order of any $R_{i}$ at $a_{i}$.

Lemma 6.4. Let $z^{\prime} \in \partial \Delta$ be a branch point. At each point of $\pi^{-1}\left(z^{\prime}\right)$, the ramification order of $\pi$ is at least 3 .

Proof. We first prove that there is no point in $\pi^{-1}\left(z^{\prime}\right)$ having the ramification order 1. Suppose there are $r$ such points, denoted by $v_{1}, \ldots, v_{r}$. We have $r<p$ because $z^{\prime}$ is a branch point. At each $v_{j}$, there is a small open neighborhood $U_{j}$ in $V$ such that $U_{j}$ is the graph of some branch of the holomorphic map $z \mapsto\left(f_{1}(z), \ldots, f_{p}(z)\right)$. At each $v_{j}$, there is one and only one component function taking a unimodular value (cf. Lemma 6.1). Therefore at most $r$ component functions can take unimodular values at the points $v_{1}, \ldots, v_{r}$. On the other hand, for each fixed $i, f_{i}$ has $r$ simple roots for its defining equation (4.2) when $z=z^{\prime}$. If $r$ is odd, Corollary 4.7 implies that at least one of these roots is unimodular and that means that every $f_{i}$ takes a unimodular value at some $v_{j}$ and this is a contradiction. If $r$ is even, Corollary 4.7 then implies that an even number of these roots are unimodular. But from Lemma 6.2, over any $z^{\prime}$, each $f_{i}$ can only take one unimodular value; therefore all the simple roots are non-unimodular and that means at each $v_{j}$ no component function takes a unimodular value. This is again a contradiction. Thus, we conclude that there is no point in $\pi^{-1}\left(z^{\prime}\right)$ having the ramification order 1 . 
Next, we argue that there is no point in $\pi^{-1}\left(z^{\prime}\right)$ having the ramification order 2. Suppose $v \in \pi^{-1}\left(z^{\prime}\right), v=\left(z^{\prime}, a_{1}, \ldots, a_{p}\right)$ is a point with a ramification order 2. By definition, the ramification of $R_{i}$ at $a_{i}$ is 2 for every $i$. We claim that this implies that $a_{i}$ is non-unimodular for every $i$. For if $R_{i}$ has a ramification order 2 at a point on the unit circle, then the unit circle will not be mapped injectively by $R_{i}$ and it contradicts Lemma 6.2. Thus, all $f_{i}$ are non-unimodular at $v$ and hence non-unimodular in a neighborhood $W$ of $v$. We can always find a point $w \in W$ such that $\pi(w)$ is not a branch point, and the fact that no $f_{i}$ takes a unimodular value at $w$ contradicts Lemma 6.1

Theorem 6.5. Let $p$ be an odd number. If $F: \Delta \longrightarrow \Delta^{p}$ is an isometric embedding with the isometric constant $k=1$ and with the sheeting number $n$ equal to $p$, then up to reparametrizations, $F$ is the $p$-th root embedding.

Proof. Fix a component function, say $f_{1}$. Let $\left\{z_{1}, \ldots, z_{m}\right\}$ be the branch points and $\left\{b_{1}, \ldots, b_{m}\right\}$ be their branching orders respectively. Then

$$
\sum_{j=1}^{m} b_{j}=2(p-1) \text {. }
$$

But $b_{j}=p-r_{j}$, where $r_{j}$ is the number of distinct values of $f_{1}\left(z_{j}\right)$. Since the multiplicity of each of these distinct values is at least 3 , we must have $r_{j} \leq p / 3$. Then

$$
\begin{gathered}
2(p-1)=\sum_{j=1}^{m} b_{j}=\sum_{j=1}^{m}\left(p-r_{j}\right) \geq \sum_{j=1}^{m} \frac{2 p}{3}=\frac{2 m p}{3} \\
\Longrightarrow \frac{3(p-1)}{p} \geq m .
\end{gathered}
$$

So $m=1,2$. But $m$ is at least 2, so by Lemma 4.9. up to some reparametrization, $f_{1}$ is a component function of the $p$-th root embedding. This is true for every $f_{i}$ and since all $f_{i}$ have the same branch points it is clear from the proof of Lemma 4.9 that there exists a single reparametrization such that every $f_{i}$ will become one of the component functions of the $p$-th root embedding. Note that if $i \neq j$, then $f_{i} \neq f_{j}$ because on each branch of $F$, only one component function can take the value $\infty$ at infinity. So the component functions of $F$ are precisely the $p$ component functions of the $p$-th root embedding.

6.2. On the maximal case. Let $p \in \mathbb{N}^{+}$and $F: \Delta \longrightarrow \Delta^{p}, F(z)=\left(f_{1}(z), \ldots\right.$, $\left.f_{p}(z)\right)$ be an isometric embedding with the isometric constant $k=1$.

Lemma 6.6. Let $r, s$ be two positive integers and $r \leq s$. Then the equation

$$
\sum_{l=1}^{r} 2^{t_{l}}=\sum_{l=1}^{s} 2^{l-1}, \quad 0 \leq t_{1} \leq t_{2} \leq \cdots \leq t_{r}
$$

has an integral solution if and only if $r=s$ and the solution is unique, i.e. $t_{l}=l-1$.

Proof. By induction on $s$.

Lemma 6.7. Let $g: \Delta \longrightarrow \Delta$ be a component function of an isometric embedding of $\Delta$ into $\Delta^{p}$. If the sheeting number of $g$ is 2 , then there exists an analytic function $h: \Delta \longrightarrow \Delta$ such that $F=(g, h)$ is an isometric embedding of $\Delta$ into $\Delta^{2}$ with the isometric constant $k=1$. 
Proof. If the sheeting number of $g$ is 2, then the Riemann-Hurwitz formula implies that $g$ has exactly two branch points; and therefore after some reparametrization $g \rightarrow g^{\alpha}, g^{\alpha}$ is one of two component functions of the square-root embedding and the lemma follows.

Theorem 6.8. Let $F: \Delta \longrightarrow \Delta^{p}, F(z)=\left(f_{1}(z), \ldots, f_{p}(z)\right)$ be an isometric embedding with the isometric constant $k=1$. For all $i$, let $s_{i}$ be the sheeting number of $f_{i}$. Assume that $s_{1} \leq s_{2} \leq \cdots \leq s_{p}$. If $s_{p}=2^{p-1}$, then the embedding can be factorized as $F=F_{p-1} \circ F_{p-2} \circ \cdots \circ F_{1}$, where each intermediate map $F_{i}: \Delta^{i} \longrightarrow$ $\Delta^{i+1}$ is given by $F_{i}\left(z_{1}, \ldots, z_{i}\right)=\left(z_{1}, \ldots, z_{i-1}, \alpha_{i}\left(z_{i}\right), \beta_{i}\left(z_{i}\right)\right)$ and $z \mapsto\left(\alpha_{i}(z), \beta_{i}(z)\right)$ is some isometric embedding of $\Delta$ into $\Delta^{2}$ with the isometric constant equal to 1 . In other words, we can write

$$
\begin{aligned}
f_{1} & =\alpha_{1}, \\
f_{2} & =\alpha_{2} \circ \beta_{1}, \\
f_{3} & =\alpha_{3} \circ \beta_{2} \circ \beta_{1}, \\
& \vdots \\
f_{p-1} & =\alpha_{p-1} \circ \beta_{p-2} \circ \cdots \circ \beta_{1}, \\
f_{p} & =\beta_{p-1} \circ \beta_{p-2} \circ \cdots \circ \beta_{1},
\end{aligned}
$$

where for all $i, z \mapsto\left(\alpha_{i}(z), \beta_{i}(z)\right)$ is an isometric embedding of $\Delta$ into $\Delta^{2}$.

Proof. First note that since every $s_{i}$ is a factor of $n$ and $n \leq 2^{p-1}$, we must have $n=2^{p-1}$ and every $s_{i}$ is a power of 2 with a power less than or equal to $p-1$. Let $r_{i}=n / s_{i}=2^{u_{i}}$. By assumption, $r_{p}=1$ and from Proposition 5.3 we have $\sum_{i=1}^{p} n / s_{i}=n$; thus,

$$
\sum_{i=1}^{p-1} 2^{u_{i}}=2^{p-1}-1=\sum_{i=1}^{p-1} 2^{i-1}, \quad 0 \leq u_{p-1} \leq u_{p-2} \leq \cdots \leq u_{1} .
$$

Therefore by Lemma 6.6,

$$
s_{j}=2^{i}, i=1, \ldots, p-1 .
$$

We prove the theorem by induction on $p$. It is trivial for $p=2$. Suppose the theorem has been proven for $\Delta^{p-1}$. Letting $\alpha_{1}=f_{1}$, since the sheeting number of $\alpha_{1}$ is 2 , by Lemma 6.7 we can find an analytic function $\beta_{1}$ such that $z \mapsto\left(\alpha_{1}(z), \beta_{1}(z)\right)$ is an isometric embedding of $\Delta$ into $\Delta^{2}$, satisfying $\left(1-\left|\alpha_{1}\right|^{2}\right)\left(1-\left|\beta_{1}\right|^{2}\right)=1-|z|^{2}$. Hence from the functional equation, we have

$$
\prod_{i=2}^{p}\left(1-\left|f_{i}\right|^{2}\right)=1-\left|\beta_{1}\right|^{2}
$$

Since 0 is not a branch point, there exists a local inverse $\beta_{1}^{-1}: U \subset \Delta \longrightarrow \Delta$ of $\beta_{1}$. Then

$$
\prod_{i=2}^{p}\left(1-\left|f_{i} \circ \beta_{1}^{-1}(z)\right|^{2}\right)=1-|z|^{2}
$$

for $z \in U$. So we see that $\left(f_{2} \circ \beta_{1}^{-1}, \ldots, f_{p} \circ \beta_{1}^{-1}\right)$ constitutes an isometric embedding of $U$ into $\Delta^{p-1}$. We already have known that such an embedding can always be extended to the whole unit disk and we let $G(z)=\left(g_{2}, \ldots, g_{p}\right)$ be the extension. For $2 \leq i \leq p$, we have $f_{i} \circ \beta_{1}^{-1}=g_{i}$ and hence $f_{i}=g_{i} \circ \beta_{1}$ on some open set. 
Thus, we have for the local inverses, $f_{i}^{-1}=\beta_{1}^{-1} \circ g_{i}^{-1}$. Recall that each of these local inverses is just the restriction of some rational function on an open set. Since the sheeting numbers of $f_{i}^{-1}, \beta_{1}^{-1}$ are $2^{i}, 2$ respectively, we deduce that the sheeting number of $g_{i}^{-1}$ is $2^{i-1}$. Therefore by the induction assumption, we can write

$$
\begin{aligned}
g_{2} & =\alpha_{2}, \\
g_{3} & =\alpha_{3} \circ \beta_{2}, \\
& \vdots \\
g_{p-1} & =\alpha_{p-1} \circ \beta_{p-2} \circ \cdots \circ \beta_{2}, \\
g_{p} & =\beta_{p-1} \circ \beta_{p-2} \circ \cdots \circ \beta_{2},
\end{aligned}
$$

where $z \mapsto\left(\alpha_{i}(z), \beta_{i}(z)\right), 2 \leq i \leq p$, are isometric embeddings of $\Delta$ into $\Delta^{2}$ with the isometric constant equal to 1 . Since $f_{1}=\alpha_{1}$ and $f_{i}=g_{i} \circ \beta_{1}$ for $2 \leq i \leq p$, the proof is complete.

\section{Classification FOR $\Delta^{2}$}

For isometric embeddings of $\Delta$ into $\Delta^{2}$, we have already known that there are only the diagonal embedding and its reparametrizations when the isometric constant $k=2$. So the only interesting case is where $k=1$.

Theorem 7.1. Let $F: \Delta \longrightarrow \Delta^{2}$ be an isometric embedding with the isometric constant $k=1$. Then up to reparametrizations, $F$ is the square root embedding.

Proof. Let $F(z)=\left(f_{1}(z), f_{2}(z)\right)$. By Proposition 5.2 and Corollary 5.4, the sheeting number must be 2. It then follows from Proposition 5.3 that the sheeting numbers of $f_{1}$ and $f_{2}$ are both 2. So from the Riemann-Hurwitz formula (cf. (4.4)), both $f_{1}$ and $f_{2}$ have two branch points. Then Lemma 4.9 says that after some reparametrization we can take $f_{1}$ to be one of the two component functions of the square root embedding. But once $f_{1}$ is known, $f_{2}$ is also uniquely determined (up to a constant factor of modulus 1 ) and therefore up to reparametrizations, $F$ is the square root embedding.

\section{Classification for $\Delta^{3}$}

The classification of isometric embeddings of $\Delta$ into $\Delta^{3}$ can be obtained from Theorem 6.5 and Theorem 6.8. By Proposition 5.2 and Corollary [5.4, if the isometric constant is $k$, then $\frac{3}{k} \leq n \leq 4$, where $n$ is the sheeting number of $V$ relative to $\pi$. For $k=3$, there are only the diagonal embedding and its reparametrizations. We first consider the case where $k=1$.

Theorem 8.1. Let $F: \Delta \longrightarrow \Delta^{3}$ be an isometric embedding with the isometric constant $k=1$. Then up to reparametrizations, $F$ is either the cube root embedding or $F$ can be factorized as $F=F_{2} \circ F_{1}$, where $F_{1}: \Delta \longrightarrow \Delta^{2}, F_{1}(z)=\left(\alpha_{1}(z), \beta_{1}(z)\right)$ is an isometric embedding with the isometric constant equal to 1 and $F_{2}: \Delta^{2} \longrightarrow$ $\Delta^{3}, F_{2}\left(z_{1}, z_{2}\right)=\left(z_{1}, \alpha_{2}\left(z_{2}\right), \beta_{2}\left(z_{2}\right)\right)$, in which $z \mapsto\left(\alpha_{2}(z), \beta_{2}(z)\right)$ is an isometric embedding of $\Delta$ into $\Delta^{2}$ with the isometric constant equal to 1 .

Proof. As $k=1$, we have $3 \leq n \leq 4$. For $n=3$, the result follows from Theorem 6.5. For $n=4$, by Proposition 5.3 there exists one $f_{i}$ with a sheeting number equal to 4 because the only solution (up to permutations) to (5.1) is $\frac{1}{2}+\frac{1}{4}+\frac{1}{4}=1$. The result then follows from Theorem 6.8. 
Next, when $k=2$, we have

Theorem 8.2. Let $F: \Delta \longrightarrow \Delta^{3}$ be an isometric embedding with the isometric constant $k=2$. Then up to reparametrizations, $F(z)=(z, \alpha(z), \beta(z))$, where $z \mapsto(\alpha(z), \beta(z))$ is an isometric embedding of $\Delta$ into $\Delta^{2}$ with the isometric constant equal to 1 .

Proof. As $k=2$, we have $2 \leq n \leq 4$. For $n=2$, the only solution (up to permutations) to (5.1) is $\frac{1}{1}+\frac{1}{2}+\frac{1}{2}=2$. So the sheeting numbers of $f_{1}, f_{2}, f_{3}$ are $1,2,2$ respectively. It is then clear that $f_{1}(z)=\gamma z$ for some $|\gamma| \in \mathbb{C}$ with $|\gamma|=1$, and we may take $\gamma=1$. Thus, the functional equation reduces to

$$
\left(1-\left|f_{2}\right|^{2}\right)\left(1-\left|f_{3}\right|^{2}\right)=1-|z|^{2},
$$

and therefore $f_{2}(z), f_{3}(z)$ constitute an isometric embedding from $\Delta$ into $\Delta^{2}$ with the isometric constant equal to 1. For $n=3$, there is no solution for (5.1). For $n=4$, the only solution (up to permutation) is $\frac{1}{1}+\frac{1}{2}+\frac{1}{2}=2$ and the sheeting numbers of $f_{1}, f_{2}, f_{3}$ are $1,2,2$ respectively and it is the same as the case when $n=2$ and hence is not possible.

\section{ISOMETRIC EMBEDDINGS BETWEEN POLYDISKS}

In this section, we will consider isometric embeddings of $\Delta^{q}$ into $\Delta^{p}$, where $q \leq p$. The polydisks will be equipped with product Poincaré metrics. We will show that all these embeddings "split", in the sense that they are induced from isometric embeddings of the unit disk into polydisks.

Let $F: \Delta^{q} \longrightarrow \Delta^{p}, F\left(z_{1}, \ldots, z_{q}\right)=\left(f_{1}\left(z_{1}, \ldots, z_{q}\right), \ldots, f_{p}\left(z_{1}, \ldots, z_{q}\right)\right)$ be an isometric embedding with the isometric constant $k$. Assume that $F(\mathbf{0})=\mathbf{0}$. Then it satisfies the functional equation

$$
\prod_{i=1}^{p}\left(1-\left|f_{1}\left(z_{1}, \ldots, z_{q}\right)\right|^{2}\right)=\prod_{j=1}^{q}\left(1-\left|z_{j}\right|^{2}\right)^{k} .
$$

The isometric constant $k$ is necessarily an integer. It can be argued in the same way as in the case of the unit disk (cf. Proposition 2.2).

We now fix $z_{2}, \ldots, z_{q}$ and consider (9.1) as a functional equation in $z_{1}$. By taking $-\sqrt{-1} \partial \bar{\partial} \log$ on both sides (with respect to $z_{1}$ only), we see that $z_{1} \mapsto F\left(z_{1}, \ldots, z_{q}\right.$ ) is an isometric embedding of $\Delta$ into $\Delta^{p}$ for every $\left(z_{2}, \ldots, z_{q}\right) \in \Delta^{q-1}$. Except when $z_{2}=\cdots=z_{q}=0$, the embedding does not preserve the origin.

Lemma 9.1. For each $i$, if $\frac{\partial f_{i}}{\partial z_{1}}$ is not constantly zero, then

$$
f_{i}\left(0, z_{2}, \ldots, z_{q}\right)=0
$$

for all $\left(z_{2}, \ldots, z_{q}\right) \in \Delta^{q-1}$.

Proof. Fixing $\left(z_{2}, \ldots, z_{q}\right) \in \Delta^{q-1}$, by following the same reasoning leading to Lemma 4.4 we see that for each $i, f_{i}\left(z_{1}, z_{2}, \ldots, z_{q}\right)$ (as a multi-valued function of $z_{1}$ ) can only take the value infinity $\infty$ at $z_{1}=\infty$. It is clear from the functional equation that $f_{i}$ as a function of $z_{1}$ satisfies the Schwarz reflection principle (cf. Proposition 4.5); therefore $f_{i}\left(0, z_{2}, \ldots, z_{q}\right)=0$. 
Lemma 9.2. Let $W \subset \mathbb{C}$ be an open set and $\left(f_{w ; 1}(z), f_{w ; 2}(z), \ldots, f_{w ; p}(z)\right)$ be a complex analytic family of holomorphic functions on $\Delta$ with $w \in W$ being the parameter such that

$$
\prod_{i=1}^{p}\left(1-\left|f_{w ; i}\right|^{2}\right)=\left(1-|z|^{2}\right)^{k}
$$

Then for all $i, \frac{\partial f_{w ; i}}{\partial w} \equiv 0$.

Proof. We can determine the algebraic subvariety extending the embedding for every $w$ in the same way as in Proposition 4.2. But from Lemma 4.1 we see that the unitary matrix required is independent of $w$ and therefore the algebraic subvariety hence obtained is also independent of $w$. It then follows that the embedding does not depend on $w$.

Theorem 9.3. Let $F: \Delta^{q} \longrightarrow \Delta^{p}, F=\left(f_{1}, \ldots, f_{p}\right)$ be an isometric embedding with the isometric constant $k$. Then after rearranging the component indices if necessary, there exist positive integers $p_{1}, \ldots, p_{q}$ with $p_{1}+\cdots+p_{q}=p$ such that

$$
\begin{array}{cc}
f_{1}, \ldots, f_{p_{1}} & \text { depend on } z_{1} \text { only; } \\
f_{p_{1}+1}, \ldots, f_{p_{1}+p_{2}} & \text { depend on } z_{2} \text { only; } \\
\vdots & \vdots \\
f_{p_{1}+\cdots+p_{q-1}+1}, \ldots, f_{p} & \text { depend on } z_{q} \text { only. }
\end{array}
$$

In particular, the $i$-th group of the above $q$ groups of functions constitutes an isometric embedding of $\Delta$ into $\Delta^{p_{i}}$ with the isometric constant equal to $k$.

Proof. By Lemma 9.1, if $\frac{\partial f_{i}}{\partial z_{1}}$ is not constantly zero, then $f_{i}\left(0, z_{2}, \ldots, z_{q}\right)=0$, $\forall\left(z_{2}, \ldots, z_{q}\right) \in \Delta^{q-1}$. From the functional equation (9.1), we see that this cannot happen to all $f_{i}$. Without loss of generality, we may assume that $\frac{\partial f_{i}}{\partial z_{1}} \equiv 0, p_{1}+$ $1 \leq i \leq p$, for some positive integer $p_{1}$. Then we can write $f_{i}\left(z_{1}, z_{2}, \ldots, z_{q}\right)=$ $f_{i}\left(z_{2}, \ldots, z_{q}\right)$, for $p_{1}+1 \leq i \leq p$. If we substitute $z_{1}=0$ in (9.1), we get

$$
\prod_{i=p_{1}+1}^{p}\left(1-\left|f_{i}\left(z_{2}, \ldots, z_{q}\right)\right|^{2}\right)=\prod_{j=2}^{q}\left(1-\left|z_{j}\right|^{2}\right)^{k} .
$$

Dividing (9.1) by (9.2), we obtain

$$
\prod_{i=1}^{p_{1}}\left(1-\left|f_{i}\left(z_{1}, z_{2}, \ldots, z_{q}\right)\right|^{2}\right)=\left(1-\left|z_{1}\right|^{2}\right)^{k} .
$$

So we have a complex analytic family (with parameters $z_{2}, \ldots, z_{q}$ ) of functions satisfying the above functional equation in $z_{1}$ and by Lemma 0.2 , for $1 \leq i \leq p_{1}$, $f_{i}$ is a function of $z_{1}$ only. Now we can argue in the same way for the variable $z_{2}$ in (9.2). By induction, the proof is complete.

Corollary 9.4. If $F: \Delta^{q} \longrightarrow \Delta^{p}$ is an isometric embedding with the isometric constant equal to $k$, then

$$
p \geq q k .
$$


Proof. As $p_{i} \geq k$, we have

$$
p=\sum_{i=1}^{q} p_{i} \geq q k
$$

\section{ACKNOWLEDGEMENT}

This article essentially derives from the author's thesis at the University of Hong Kong, and he would like to express his gratitude to his supervisor, Prof. Ngaiming Mok, for his inspiring guidance and encouragement.

\section{REFERENCES}

[E.53] Calabi, E., Isometric imbedding of complex manifolds, Ann. of Math. (2) 58 (1953), no. 2, 1-23. MR0057000 (15:160c)

[LE03] Clozel, L. and Ullmo, E., Correspondances modulaires et mesures invariantes, J. Reine Angew. Math. 558 (2003), 47-83. MR1979182(2005a:11056)

[N.a] Mok, N., Erratum to "Local holomorphic isometric embeddings arising from correspondences in the rank-1 case", 2005, http://hkumath.hku.hk/ nmok/Erratum.pdf.

[N.b] Local holomorphic isometric embeddings arising from correspondences in the rank-1 case, Contemporary Trends in Algebraic Geometry and Algebraic Topology (Chern, S.S., Fu, L., and Hain, R., eds.), Nankai Tracts in Mathematics, Vol. 5, World Scientific, New Jersey, 2002, pp. 155-166. MR1945359 (2003k:32032)

[N.ng] _ Extension of germs of holomorphic isometries up to normalization constants with respect to the Bergman metric, preprint, http://hkumath.hku.hk/ imr/ IMRPreprintSeries/2009/IMR2009-9.pdf.

Department of Mathematics, The University of Hong Kong, Pokfulam Road, Hong KONG

E-mail address: math.scng@hku.hk 